\title{
Temperature-Dependent Variation in Self-Reported Contagious Yawning
}

\author{
Omar Tonsi Eldakar • Melissa Dauzonne • \\ Yana Prilutzkaya • Daniel Garcia • Carolyn Thadal • \\ Andrew C. Gallup
}

Received: 19 January 2015 / Revised: 20 February 2015 / Accepted: 24 February 2015 /

Published online: 5 March 2015

(C) Springer International Publishing 2015

\begin{abstract}
The thermoregulatory theory posits that yawns function to cool the brain in part due to counter-current heat exchange with the deep inhalation of ambient air. In support of this theory, previous cross-cultural research on humans has shown that self-reported contagious yawning frequency varies between seasons with distinct ambient temperature ranges. However, it remains possible that differences in yawning across seasons are a result of physiological circadian changes across the year rather than variation in ambient temperature. In an attempt to address this question, here we discuss the results of a study investigating the variation in the frequency of self-reported contagious yawning within a restricted range of a single season in one geographic location. A total of 142 pedestrians were recruited outdoors during an 18-day period over the summer in an equatorial monsoon climate in southern Florida, USA. Consistent with the thermoregulatory theory of yawning, results showed that self-reported contagious yawning frequency varied predictably across temperature gradients. This was true after statistically controlling for relative humidity, time of day, time spent outside, testing day, age of participant, and amount of sleep the night before. These findings provide further evidence suggesting a brain cooling function to yawning.
\end{abstract}

Keywords Yawning - Brain temperature - Ambient temperature - Thermoregulation · Contagious yawning $\cdot$ Climate variation

\section{Introduction}

Yawning is characterized by a large gaping of the mouth with a deep inspiration, a brief acme period with peak muscle contraction, and passive closure of the jaw with a shorter

O. T. Eldakar $(\bowtie) \cdot$ M. Dauzonne $\cdot$ Y. Prilutzkaya $\cdot$ D. Garcia $\cdot$ C. Thadal

Department of Biology, Nova Southeastern University, Fort Lauderdale, FL, USA

e-mail: oeldakar@gmail.com

A. C. Gallup ( $\square)$

Psychology Department, State University of New York at Oneonta, Oneonta, NY, USA

e-mail: a.c.gallup@gmail.com 
expiration of air (Barbizet 1958). This behavior appears highly conserved, with reported observations of yawns having been documented across vertebrate classes (Craemer 1924; Luttenberger 1975; Baenninger 1987; Sauer and Sauer 1967). Over time numerous functions of yawning have been suggested (Smith 1999), ranging from regulating middle ear pressure (Laskiewicz 1953) to preventing lung atelectasis (Cahill 1978). While many hypotheses have been proposed for why we yawn, few have been empirically supported (Gallup 2011). For example, it is commonly believed that we yawn to increase oxygen in the blood, yet this respiratory hypothesis was tested and falsified over 25 years ago (Provine et al. 1987).

In recent years, the thermoregulatory theory has emerged as the most empirically supported physiological explanation for why we yawn (see Gallup and Eldakar 2013 for a discussion of evidence and critiques). This theory states that the motor action pattern of yawning functions as a brain cooling mechanism (Gallup and Gallup 2007, 2008). Consistent with this theory, research on both rats (Rattus norvegicus) and humans has demonstrated that yawns are surrounded by predicted fluctuations in brain and skull temperature, i.e., yawning is preceded by rises in temperature and followed by decreases in temperature thereafter (humans, Gallup and Gallup 2010; rats, ShoupKnox et al. 2010). Furthermore, research on birds (Melopsittacus undulatus) has shown that following stress-induced hyperthermia the latency to yawn is negatively correlated with the peak body temperature (Miller et al. 2010). Literature from diverse fields of medicine, physiology and pharmacology also support the connection between yawning and brain thermoregulation (Gallup and Gallup 2008).

According to the thermoregulatory theory, yawning acts to cool brain temperature through thermoregulatory mechanisms of countercurrent heat exchange, evaporative cooling and enhanced cerebral blood flow (for a discussion, see Gallup and Hack 2011). As a result, the ambient air temperature is critical for yawning to function as a brain cooling mechanism, and therefore yawns should be constrained to a relatively narrow range of temperature, i.e., a thermal window (Gallup and Gallup 2007). The predictions of this hypothesis are that yawns should (1) increase in frequency with initial rises in ambient temperature from a thermal neutral zone, as this stimulates thermoregulatory cooling mechanisms to control temperatures within a normal range, (2) decrease as ambient temperatures approach or exceed body temperature, since yawns would be counterproductive by introducing deep inhalations of elevated temperature into the body, and likewise (3) diminish when temperatures fall below a certain point, because thermoregulatory cooling responses are no longer necessary and countercurrent heat exchange may cause deviations in internal temperature which fall below homeostasis (Massen et al. 2014). Aside from having different triggers, the motor action patterns of spontaneous and contagious yawns are indistinguishable and therefore these predictions should apply to both forms.

The thermal window hypothesis is already well supported by existing research in comparative psychology and behavioral biology. Observational research on non-human primates has documented that spontaneous yawning frequency becomes elevated in warm ambient temperatures (Campos and Fedigan 2009; Deputte 1994). Furthermore, experimental studies on birds and rats in the laboratory are consistent with the first two predictions of this model (Gallup et al. 2009, 2010, 2011). For example, in budgerigars spontaneous yawns increase when temperatures are elevated from 22 to $34 \mathrm{C}$, but then decrease in frequency when temperatures are held between 34 and 38C. Similar effects 
have been documented in Sprague Dawley rats using slightly different temperature ranges (Gallup et al. 2011).

Two recent naturalistic studies on humans have also investigated the relationship between ambient temperature and yawning frequency by surveying pedestrians while outdoors in the same geographical location during distinct seasonal climate conditions. The first study measured self-reported contagious yawning among pedestrians during the summer and winter months in the arid desert climate of Arizona, USA following exposure to a contagious yawning stimulus (Gallup and Eldakar 2011). The second study used the same methodology but measured the response of summer and winter yawning among pedestrians in the temperate climate of Vienna, Austria (Massen et al. 2014). Overall, the results from these studies demonstrated that yawning is significantly less common at temperature extremes. In particular, self-reported contagious yawning was highest in the winter in Arizona and in the summer in Vienna, when ambient temperatures were near room temperature $(19.4 \mathrm{C} ; 21.9 \mathrm{C})$, but yawning rates declined significantly in the summer months of Arizona, when temperatures averaged $37 \mathrm{C}$, and in the winter months of Vienna, when temperatures averaged 1.4C. Furthermore, both studies demonstrated that temperature was a significant predictor of yawning even when controlling for other variables associated with yawning frequency.

The aforementioned literature, including naturalistic observations and controlled laboratory experiments, suggests that underlying mechanisms controlling yawning are sensitive to ambient temperature. Despite accounting for changes in daylight cycles associated with winter and summer months, and statistically controlling for hours of sleep prior to testing, it remains possible that physiological circadian changes across the year contributed to the pattern of self-reported contagious yawning in humans during distinct seasons. Therefore, the current study was designed to investigate whether significant variation in yawning exists within a short timeframe in a single season. Using the same approach as Gallup and Eldakar (2011) and Massen et al. (2014), we recruited pedestrians outside during an 18-day period over the summer in the equatorial monsoon climate of southern Florida, USA (Kottek et al. 2006). Ambient temperatures during this period ranged from 27.8 to $36.1 \mathrm{C}$. Based on the first two predictions of the thermal window hypothesis, we expected yawns to be (1) most frequent during the lower bound of this range, since these temperatures are moderately warm and deviate from a thermal neutral zone, and (2) least frequent at the highest temperatures as these are drawing near human body temperature.

\section{Methods}

Participants

A total of 142 random pedestrians (69 male; 70 female; 3 unidentified) were recruited in the city of Ft. Lauderdale, Florida (Lat.: 26.13; Lon: 80.15) across 9 days during an 18-day period from August to September 2014. Participants were all 18 years or older and gave consent to participate in this study. The local Institutional Review Board approved this research. No financial compensation was provided for participation in this study. 


\section{Procedure}

Replicating the methods of Gallup and Eldakar (2011) and Massen et al. (2014), pedestrians were recruited by trained researchers while outdoors and asked to participate in a study about contagious yawning. Pedestrians were approached outside while in public settings (courtyards, pedestrian thoroughfares). If individuals agreed to participate, they were instructed to carefully look through a packet containing a series of 19 images of people yawning and then immediately respond to a survey related to their own yawning behavior during the experiment. This method of self-report has proven to be a valid measure of yawning in similar conditions (Greco and Baenninger 1989). Participants also provided information regarding their age, how long they slept the night before, and how long they had been outside prior to participating in this study.

Because research has shown that participants are less likely to yawn in an experimental setting when they are being observed (Baenninger and Greco 1991), the researchers immediately walked away from the participant(s) after they agreed to take the survey and intentionally avoided directing their attention toward them until it was completed. During this time, which varied between participants but typically lasted between 2 and $5 \mathrm{~min}$, the experimenters would record the local ambient temperature and relative humidity (\%) at the time of participation using a digital thermometer/ hygrometer positioned in the shade. All data were collected between 11:30 and 16:00 h.

\section{Analysis}

A binary logistic regression was used to assess the influence of the independent variables ambient temperature (Celsius), relative humidity (\%), time of day (100 h), time spent outside prior to being tested (minutes), testing day (1-9, chronological), age (years), and sleep the night before (hours) on self-reported contagious yawning frequency (dependent variable: yes/no), with Nagelkerke $R^{2}$ being reported for significant results. The same model was also used to assess how these variables influenced the reported urge to yawn among pedestrians that did not report yawning during the survey.

\section{Results}

Overall, 53 participants $(37.3 \%)$ reported yawning contagiously at least once while either viewing the stimulus images or responding to the following survey questions, which is consistent with previous studies (30.0 to $34.4 \%$; Gallup and Eldakar 2011; Massen et al. 2014). There was no difference in the reported incidence of yawning between males and females $\left(33.3 \%\right.$ vs. $\left.38.6 \% ; \chi^{2}(1)=0.414, p=0.520\right)$. Independent variables in our analysis included ambient temperature, relative humidity, time of day, time spent outside prior to testing, testing day, age of the participant, and hours of sleep reported the previous night (descriptive statistics: Table 1). A binary logistic regression was used to assess how these variables influenced whether a participant yawned (yes/ no) during the survey. This model revealed that temperature was the only significant predictor $\left(R^{2}=0.212\right.$; temperature: $\beta=-0.486, p<0.001$; Table 2$)$. In other words, the self-reported yawning is quite high at moderately warm ambient temperatures but then decreases as ambient temperatures approached body temperature, even when 
Table 1 Descriptive statistics of independent variables

\begin{tabular}{lrrrrrr}
\hline & N & Range & Minimum & Maximum & Mean & Std. Dev. \\
\hline Temperature (C) & 142 & 8.33 & 27.78 & 36.11 & 33.142 & 1.758 \\
Relative Humidity (\%) & 142 & 37.00 & 38.00 & 75.00 & 55.007 & 8.785 \\
Time of day (100 h) & 136 & 4.50 & 11.50 & 16.00 & 13.550 & 1.101 \\
Time Outside (Min) & 142 & 570.00 & 0.00 & 570.00 & 89.891 & 117.483 \\
Testing day (1-9) & 142 & 8.00 & 1.00 & 9.00 & 4.232 & 2.649 \\
Age (years) & 137 & 52.00 & 18.00 & 70.00 & 29.540 & 14.803 \\
Sleep (hours) & 138 & 7.00 & 3.00 & 10.00 & 6.873 & 1.340 \\
\hline
\end{tabular}

accounting for other variables. Figure 1 depicts this pattern and provides a reference line to the self-reported yawning frequency observed in similar naturalistic studies at room temperature (19.4C; 21.9C) (Gallup and Eldakar 2011; Massen et al. 2014). The same regression was also used for the self-reported urge to yawn (yes/no) among pedestrians that did not report yawning during the survey. This model revealed the same basic findings with temperature as the only significant predictor $\left(R^{2}=0.111\right.$; temperature: $\beta=-0.416, p=0.038$; Table 3 ), i.e., among pedestrians that did not yawn during the study, there was a negative relationship between the self-reported urge to yawn and ambient temperature.

\section{Discussion}

The thermoregulatory theory states that the physiological consequences of yawning function in brain cooling. Accordingly, the thermal window hypothesis, an extension of this theory, states that the expression of yawning should be sensitive to ambient temperature variation. Unlike previous studies to investigate the relationship between ambient temperature and yawning frequency in humans, which have sampled pedestrians outside during distinct seasonal conditions and daylight cycles, the current study controlled for potential physiological changes that could emerge from changes in daylight cycles throughout the year by restricting data acquisition across a short timeframe. Consistent with the thermal window hypothesis of the thermoregulatory

Table 2 Binary logistic regression (Yawn: yes/no)

\begin{tabular}{llllrl}
\hline Yawn (yes/no) & B & S.E. & Wald & df & Sig. \\
\hline Temperature (C) & -0.486 & 0.137 & 12.483 & 1 & 0.000 \\
Relative Humidity (\%) & -0.044 & 0.034 & 1.637 & 1 & 0.201 \\
Time of day (100 h) & 0.219 & 0.235 & 0.871 & 1 & 0.351 \\
Time Outside (Min) & 0.001 & 0.002 & 0.091 & 1 & 0.763 \\
Testing day (1-9) & 0.008 & 0.115 & 0.005 & 1 & 0.942 \\
Age (years) & -0.005 & 0.020 & 0.059 & 1 & 0.807 \\
Sleep (hours) & -0.036 & 0.158 & 0.053 & 1 & 0.819
\end{tabular}




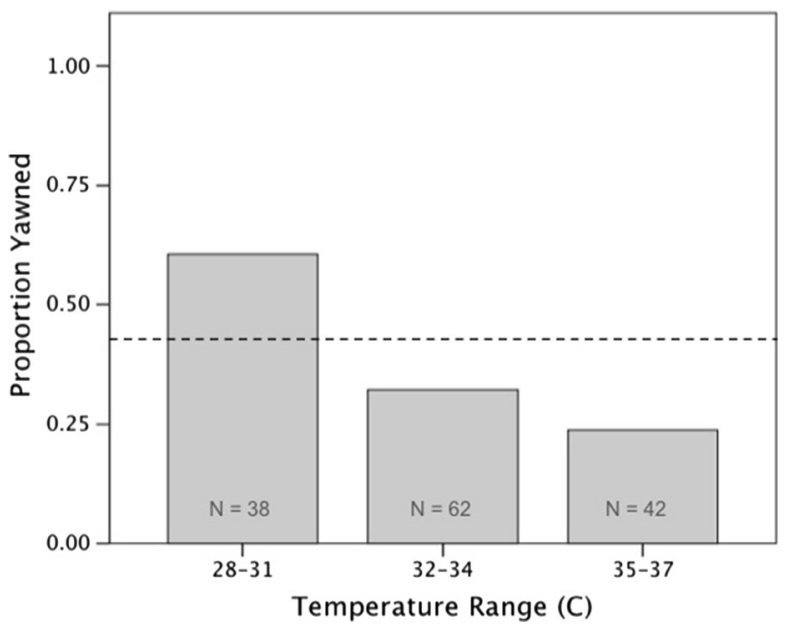

Fig. 1 Self-reported yawning declined across ambient temperature gradients. The sample size is provided for each temperature range, and the reference line (dotted) depicts the average self-reported yawning frequency observed in similar naturalistic studies at room temperature (19.4C; 21.9C) (Gallup and Eldakar 2011; Massen et al. 2014)

theory, results showed that self-reported contagious yawning frequency varied predictably across ambient temperature gradients. That is, there was an increase in selfreported contagious yawning in moderately warm conditions deviating from room temperature, but as ambient temperature approached human body temperature pedestrians exposed to a contagious yawning stimulus were significantly less likely to report yawning. In addition, the same pattern emerged for the self-reported urge to yawn among pedestrians that did not yawn while being tested.

This study demonstrates within season variation in self-reported contagious yawning is largely explained by deviations in ambient temperature. These findings are present even after statistically controlling for relative humidity, time of day, time spent outside prior to being surveyed, testing day across the two and half week period, age of the participant, and hours of sleep the night before. Overall, these results support the interpretations of previous naturalistic reports on human yawning, and provide further evidence suggesting that the underlying mechanisms controlling the expression of yawning are involved in thermoregulatory physiology.

Table 3 Binary Logistic Regression (Urge to Yawn: yes/no)

\begin{tabular}{llllll}
\hline Urge Yawn (yes/no) & B & S.E. & Wald & df & Sig. \\
\hline Temperature (C) & -0.416 & 0.201 & 4.301 & 1 & 0.038 \\
Relative Humidity (\%) & 0.007 & 0.038 & 0.037 & 1 & 0.847 \\
Time of day (100 h) & 0.277 & 0.285 & 0.947 & 1 & 0.331 \\
Time Outside (Min) & 0.000 & 0.003 & 0.014 & 1 & 0.905 \\
Testing day (1-9) & -0.078 & 0.120 & 0.423 & 1 & 0.515 \\
Age (years) & -0.010 & 0.021 & 0.250 & 1 & 0.617 \\
Sleep (hours) & -0.043 & 0.192 & 0.050 & 1 & 0.824 \\
\hline
\end{tabular}




\section{References}

Barbizet, J. (1958). Yawning. Journal of Neurology, Neurosurgery, and Psychiatry, 21(3), 203-209.

Baenninger, R. (1987). Some comparative aspects of yawning in Betta splendens, Homo sapiens, Panthera leo, and Papio sphinx. Journal of Comparative Psychology, 101, 349-354.

Baenninger, R., \& Greco, M. (1991). Some antecedents and consequences of yawning. Psychological Record, 41(4), 453-460.

Cahill, C. A. (1978). Yawn maneuver to prevent lung atelectasis. AORN Journal, 27, 1000-1004.

Campos, F. A., \& Fedigan, L. M. (2009). Behavioral adaptations to heat stress and water scarcity in whitefaced capuchins (Cebus capucinus) in Santa Rosa National Park, Costa Rica. American Journal of Physical Anthropology, 138, 101-111.

Craemer, F. (1924). Uber sodbrnnan and gähnen. Gastroenterogia Archiven fur Verdauungskrankheiten, 33, $149-162$.

Deputte, B. L. (1994). Ethological study of yawning in primates. I. Quantitative analysis and study of causation in two species of old world monkeys (Cercocebus albigena and Macaca fascicularis). Ethology, 98, 221-245.

Gallup, A. C. (2011). Why do we yawn? Primitive versus derived features. Neuroscience \& Biobehavioral Reviews, 35, 765-769.

Gallup, A. C., \& Eldakar, O. T. (2011). Contagious yawning and seasonal climate variation. Frontiers in Evolutionary Neuroscience, 3, 1-4.

Gallup, A. C., \& Eldakar, O. T. (2013). The thermoregulatory theory of yawning: what we know from over 5 years of research. Frontiers in Neuroscience, 6, 1-13.

Gallup, A. C., \& Hack, G. D. (2011). Human paranasal sinuses and selective brain cooling: a ventilation system activated by yawning? Medical Hypotheses, 77(6), 970-973.

Gallup, A. C., \& Gallup, G. G., Jr. (2007). Yawning as a brain cooling mechanism: nasal breathing and forehead cooling diminish the incidence of contagious yawning. Evolutionary Psychology, 5, 92-101.

Gallup, A. C., \& Gallup, G. G., Jr. (2008). Yawning and thermoregulation. Physiology \& Behavior, 95(1), 1016.

Gallup, G. G., Jr., \& Gallup, A. C. (2010). Excessive yawning and thermoregulation: two case histories of chronic, debilitating bouts of yawning. Sleep and Breathing, 14(2), 157-159.

Gallup, A. C., Miller, M. L., \& Clark, A. B. (2009). Yawning and thermoregulation in budgerigars, Melopsittacus undulatus. Animal Behaviour, 77(1), 109-113.

Gallup, A. C., Miller, M. L., \& Clark, A. B. (2010). The direction and range of ambient temperature change influences yawning in budgerigars (Melopsittacus undulatus). Journal of Comparative Psychology, 124(2), 133.

Gallup, A. C., Miller, R. R., \& Clark, A. B. (2011). Changes in ambient temperature trigger yawning but not stretching in rats. Ethology, 117(2), 145-153.

Greco, M., \& Baenninger, R. (1989). Self-report as a valid measure of yawning in the laboratory. Bulletin of the Psychonomic Society, 27(1), 75-76.

Kottek, M., Grieser, J., Beck, C., Rudolf, B., \& Rubel, F. (2006). World map of the Köppen-Geiger climate classification updated. Meteorologische Zeitschrift, 15(3), 259-263.

Laskiewicz, A. (1953). Yawning with regard to the respiratory organs and the ear. Acta Oto-Laryngologica, $43,267-270$.

Luttenberger, F. (1975). On yawning in reptiles. Zeitschrift für Tierpsychologie, 37, 113-137.

Massen, J. J., Dusch, K., Eldakar, O. T., \& Gallup, A. C. (2014). A thermal window for yawning in humans: yawning as a brain cooling mechanism. Physiology \& Behavior, 130, 145-148.

Miller, M. L., Gallup, A. C., Vogel, A. R., \& Clark, A. B. (2010). Handling-stress initially inhibits, but then potentiates yawning in budgerigars (Melopsittacus undulatus). Animal Behaviour, 80, 615-619.

Provine, R. R., Tate, B. C., \& Geldmacher, L. L. (1987). Yawning: no effect of $3-5 \% \mathrm{CO}^{2}, 100 \% \mathrm{O}^{2}$, and exercise. Behavioral and Neural Biology, 48(3), 382-393.

Sauer, E. F., \& Sauer, E. M. (1967). Yawning and other maintenance activities in the South African Ostrich. The Auk, 571-587.

Shoup-Knox, M. L., Gallup, A. C., Gallup, G. G., Jr., \& McNay, E. C. (2010). Yawning and stretching predict brain temperature changes in rats: support for the thermoregulatory hypothesis. Frontiers in Evolutionary Neuroscience, 2, 1-5.

Smith, E. O. (1999). Yawning: an evolutionary perspective. Human Evolution, 14.3, 191-198. 